Unfallchirurg 2017 · 120:722-727

DOI 10.1007/s00113-017-0354-x

Online publiziert: 13. Juni 2017

(c) Der/die Autor(en) 2017. Dieser Artikel ist eine Open-Access-Publikation.

Redaktion

T. Neubauer, Horn

CrossMark

A. Antoni · T. Heinz · J. Leitgeb

Universitätsklinik für Unfallchirurgie, Medizinische Universität Wien, Wien, Österreich

\title{
Polytrauma und begleitendes Schädel-Hirn-Trauma
}

\section{Die Rolle des Unfallchirurgen}

\begin{abstract}
Das Schädel-Hirn-Trauma (SHT) und der hämorrhagische Schock sind die beiden häufigsten Todesursachen beim Polytrauma. Schon eine kurze Episode der Hypotonie verdoppelt die Mortalität des SHT. Gerade in Zeiten der zunehmenden Spezialisierung in der Medizin braucht es ein effizientes und effektives Management des Polytraumas mit begleitendem SHT, um ein gutes Behandlungsergebnis zu erzielen. Welche Voraussetzungen müssen dafür gegeben sein und wer kann sie erfüllen?
\end{abstract}

\section{Hintergrund und Fragestellung}

Eine gute präklinische Versorgung und die Entwicklung von Traumazentren haben in den vergangenen Jahrzehnten nachweislich zu einer Halbierung der Mortalität beim Polytrauma geführt. Doch auch die Standardisierung von Abläufen mit optimierten präklinischen Prozessen und Regionalisierung des Gesundheitssystems mit spezialisierten Traumazentren haben ihre Grenzen. Patienten müssen das Traumazentrum zeitnah erreichen und Behandlungsentscheidungen letztlich von Menschen getroffen und durchgeführt werden. In mitteleuropäischen Traumazentren ist üblicherweise der Unfallchirurg der sogenannte „Traumateamleader“. Während in Mitteleuropa aufgrund zunehmender Spezialisierung die Unfallchirurgie immer mehr Gefahr läuft, an Spektrum zu verlieren, entwickelte sich in den USA „acute care surgery“, eine der Unfallchirurgie verwandte Fachdisziplin. Diese „acute care surgeons“ erfüllen primär die Rolle des Stabilisierens und Priorisierens und haben im Gegensatz zur mitteleuropäischen Unfallchirurgie ein vergleichsweise kleines operatives Spektrum. In vielen Ländern stellt statt des Chirurgen auch ein Notarzt oder Anästhesist die Speerspitze des Traumateams dar.

- Welches Wissen und welche Fertig-

keiten qualifizieren für das Management des Polytraumas mit SchädelHirn-Trauma (SHT)?

- Wer soll die Verantwortung für das Management dieser Patienten tragen und wie weit soll dieses in jeweiliger Kompetenz gehen?

- Sind in Zukunft nur die Stabilisierung, Diagnostik und Vorbereitung für andere Fachdisziplinen die Aufgaben oder beinhaltet das Akutmanagement auch die operative Therapie der Patienten mit mehr oder weniger Konsultation anderer Fachdisziplinen?

Im Folgenden werden die Fragestellungen anhand von internationaler Literatur diskutiert und ein wissenschaftlich fundierter Boden für diese standespolitischen Glaubensfragen geschaffen.

\section{Ausgangslage - Mortalität und Outcome}

Das SHT ist die Haupttodesursache beim Polytrauma gefolgt vom hämorrhagischen Schock. Die Letalität des Polytraumas, definiert als Mehrfachverletzung mit einem Injury Severity Score (ISS) ab 16, liegt international zwischen 18 und $23 \%$ im Vergleich zu $37 \%$ in den 70er Jahren [14, 34, 36]. Das schwere
SHT ohne Begleitverletzungen hat eine Letalität von durchschnittlich $30 \%$ [24, 26, 28]. Der Einfluss schwerer Mehrfachverletzungen auf ein SHT zeigt sich durch die Steigerung der Letalität mit Werten bis zu $46 \%$ [26].

\section{》) Schwere Mehrfachverletzun- gen bei SHT steigern die Letalität mit Werten bis zu $46 \%$}

Die Behandlungsergebnisse von Polytrauma mit SHT sind deutlich schlechter als ohne begleitendes SHT. Eine Untersuchung von Gross et al. [21] zeigte, dass selbstständiges Leben, gemessen mit der Glasgow Outcome Scale (GOS) von 4 und 5, bei $95 \%$ der überlebenden Polytraumapatienten möglich war, während nur $71 \%$ der überlebenden Polytraumapatienten mit schwerem SHT einen GOS-Wert von 4 oder 5 erreichten. Ein hohes Patientenalter beeinflusst das Ergebnis des schweren SHT überdies negativ. In einer Metaanalyse von Hukkelhoven et al. [24] zeigte sich ab einem Alter von 55 Jahren bei der Mehrzahl der Patienten ein unzufriedenstellendes Ergebnis.

\section{Outcomefaktoren}

Als wichtigste Einflussgröße auf die Letalität des SHT beim Polytrauma zeigte sich die Hypotension, wobei schon eine Episode von systolischem Blutdruck $<90 \mathrm{~mm} \mathrm{Hg}$ die Mortalität verdoppelt [5]. Weiter beeinflusst Hypoxie das Behandlungsergebnis negativ, weshalb eine Sauerstoffsättigung $>90 \%$ und Sauerstoffpartialdruckwerte $>60 \mathrm{~mm} \mathrm{Hg}$ 


\section{Infobox 1 Therapieziele beim}

Polytrauma mit SHTa

Hier steht eine Anzeige.

- Systolischer Blutdruck >90 mm Hg

- Sauerstoffsättigung $>90 \%$

- Sauerstoffpartialdruck $>60 \mathrm{~mm} \mathrm{Hg}$

- Kranialer Perfusionsdruck (CPP)

$>70 \mathrm{~mm} \mathrm{Hg}$ (CPP = MAP - ICP)

- $<4 \mathrm{~h}$ bis zur operativen Versorgung des SHT

- Gerinnungsoptimierung/Antidot bei bekannter Antikoagulation

aZur Vermeidung sekundärer Schäden durch zerebrale Hypoperfusion mit Verschlechterung der primären Schäden und Ausbildung eines Hirnödems

SHT Schädel-Hirn-Trauma, MAP mittlerer arterieller Druck, ICP intrakranieller Druck

empfohlen werden [2]. Therapieziele bei Polytrauma mit SHT sind in • Infobox 1 aufgelistet. Ein direkter Einfluss auf das schwere SHT durch extrakranielle Verletzungen ohne begleitende hämodynamische/respiratorische Instabilität konnte bisher nicht identifiziert werden [28].

Nicht direkt beeinflussbar, aber in Therapieentscheidungen mit einzubeziehen, sind patientenbezogene Faktoren wie Alter, Begleiterkrankungen und Allgemeinzustand.

\section{"Time is brain"}

Verbesserungen im Rettungswesen haben zu einer starken Senkung der Mortalität beim Polytrauma in den vergangenen Jahrzehnten geführt. Dabei scheint der Zeitgewinn der wichtigste Parameter für das Outcome zu sein [34, 36]. Zahlreiche Studien zeigen, dass ein Zeitverlust vor der operativen Versorgung mit einer erhöhten Mortalität einhergeht. Hierbei wurde in der Literatur eine Grenze von $4 \mathrm{~h}$ als kritische Zeitmarke zwischen Unfall und operativer Versorgung angegeben $[38,39]$. Auf dieser Basis empfehlen Leitlinien und zahlreiche Autoren eine Vermeidung zeitaufwendiger Sekundärtransporte [1, 18].

\section{Traumazentrum als Lösung aller Probleme}

Die Bildung von Traumazentren und Festlegung von Kriterien für den Trans- 
Unfallchirurg 2017· 120:722-727 DOI 10.1007/s00113-017-0354-x

(c) Der/die Autor(en) 2017. Dieser Artikel ist eine Open-Access-Publikation.

\section{A. Antoni $\cdot$ T. Heinz $\cdot$ J. Leitgeb}

\section{Polytrauma und begleitendes Schädel-Hirn-Trauma. Die Rolle des Unfallchirurgen}

\section{Zusammenfassung}

Hintergrund. Das Outcome des Polytraumas ist bei begleitendem Schädel-Hirn-Trauma (SHT) mit einer erhöhten Mortalität sowie erniedrigten Lebensqualität verbunden. Dies macht eine zielgerichtete Versorgung notwendig, wobei die zunehmende Spezialisierung in der Medizin die Rolle des Unfallchirurgen für das Management dieser Patienten infrage stellen könnte.

Fragestellung. Welche Faktoren müssen beachtet werden, um ein adäquates Behandlungsergebnis des Polytraumas mit begleitendem SHT zu erreichen? Wer soll die Verantwortung für das Management dieser Patienten tragen und wie weit reicht Management?

Material und Methode. Es erfolgte eine Literaturrecherche in der Medline-Datenbank über PubMed mithilfe medizinischer Schlagwörter ("medical subject headings") sowie Freitextsuche.

Ergebnisse. Entscheidend für das Outcome zeigten sich die Vermeidung von Hypotonie, die Anwendung prä- und innerklinischer Standards mit raschem Transport in geeignete Zentren, prioritätenbezogener Diagnostik und Therapie sowie strikter Befolgung der Prinzipien der Damage Control Surgery. International werden Patienten mit Polytrauma und SHT, bedingt durch die jeweiligen Traumasysteme, geographischen Gegebenheiten und vorhandenen Ressourcen, von unterschiedlichen Fachdisziplinen behandelt. Vergleiche der operativen und konservativen Versorgung durch unterschiedliche Fachdisziplinen zeigten jedenfalls vergleichbare Ergebnisse.
Schlussfolgerungen. Auch im Zeitalter der Standardisierung und des hohen Spezialisierungsgrades in der Medizin scheint der Unfallchirurg eine optimale Versorgung des Polytraumas mit begleitendem SHT erfüllen zu können. Das Hauptaugenmerk liegt hierbei auf der Sicherstellung der prioritätenbezogenen Diagnostik und Therapie unter Einhaltung von Prinzipien der Damage Control Surgery.

Schlüsselwörter

Standardisierung $\cdot$ Spezialisierungsgrad

Outcome - Damage Control Surgery .

Traumasystem

\section{Polytrauma and concomitant traumatic brain injury. The role of the trauma surgeon}

\section{Abstract}

Background. Concomitant traumatic brain injury (TBI) increases mortality and reduces quality of life of polytrauma patients. These facts demand effective treatment strategies while the growing specialization of medicine is questioning the role of the trauma surgeon in the management of these patients.

Objectives. Which factors influence outcome of polytrauma with concomitant TBI? Who should be responsible for the management of these patients and what is the limit of management?

Materials and methods. A literature search using Medline via PubMed was performed with Medical Subject Headings and text word search.

Results. The crucial factors for outcome are absence of hypotension, adherence to pre- and in-hospital standards like fast transportation to appropriate centers, prioritybased diagnostic and therapeutic strategies and strict adherence to principles of damage control surgery. Patients with polytrauma and $\mathrm{TBI}$ are treated by different specialties around the world based on the trauma system, geographic circumstances and resources. Investigations of operative and conservative management by different medical specialties showed comparable outcomes.

Conclusions. In an age of standardization and a high degree of specialization in the field of medicine, the trauma surgeon still seems to be able to ensure an optimal treatment of polytrauma and concomitant TBI by focusing on priority-based diagnostic and therapeutic strategies and adhering to principles of damage control surgery.

Keywords

Standardization · Specialization · Outcome · Damage control surgery · Trauma care system port in ein solches hat erwiesenermaßen zur Mortalitätsreduktion Schwerverletzter beigetragen [22]. Sekundärtransporte und damit Zeitverlust bis zur operativen Versorgung konnten durch eine solche Regionalisierung der Versorgung und Bildung von Netzwerken verringert werden [4, 22, 36]. Die Definition von Traumazentren mit entsprechenden Levels ist in • Tab. 1 aufgeführt.

Im Falle des Polytraumas mit SHT wird durch die Anwendung regionaler Kriterien meist ein direkter Transport in ein Level-I-Traumazentrum angestrebt [18]. Dort sollten definitionsgemäß Neu- rochirurgen zu jeder Zeit verfügbar sein. Doch die Realität zeigt, dass nicht alle Polytraumapatienten in ein Zentrum der Maximalversorgung transportiert werden können [30] und Neurochirurgen nicht immer verfügbar sind [37]. Dies kann neben Ressourcenknappheit durch direkte Hindernisse für den Transport bedingt sein, wie geographische $\mathrm{Be}$ dingungen oder Witterungseinflüsse im Falle der Notwendigkeit eines luftgebundenen Transports.

\section{Prioritäten setzen}

Durch die Verbreitung von Advanced Trauma Life Support (ATLS) ist das standardisierte Management von Polytraumatisierten in Traumazentren ein globaler Standard geworden. Der positive Effekt der Standardisierung der Abläufe und Rollenverteilung in Traumateams konnte in zahlreichen Studien gezeigt werden $[4,8,36]$. Insbesondere gelang eine Reduktion der frühen Mortalität durch hämorrhagischen Schock [10]. Die Behandlung von Schwerverletzten in Traumazentren wird inzwischen 


\section{Tab. 1 Definition der Levels von Traumazentren des American College of Surgeons [7]}

Level I Maximalversorgung, alle Verletzungen können definitiv versorgt werden, inklusive Rehabilitation

Level II Äquivalent zu Level I mit Ausnahme hochkomplexer Versorgungen (z. B. Replantationen)

Level III Akutversorgung mit Stabilisierung und definitiver Versorgung von Fällen geringerer Komplexität, System für Transfer an übergeordnetes Zentrum

Level IV Advanced Trauma Life Support, Stabilisierung, System für Transfer an übergeordnetes Zentrum

weltweit durch Traumateams mit festgelegtem Traumateamleader geführt. Die Rolle des Traumateamleaders besteht darin, primär den Überblick über die Gesamtsituation des Patienten zu bewahren und prioritätenbezogene Diagnostik und Therapie einzuleiten.

In Mitteleuropa ist traditionell der Unfallchirurg Traumateamleader. International teilen sich Notärzte, Allgemeinchirurgen, Anästhesisten und Unfallchirurgen die Rolle des Traumateamleaders. Untersuchungen zu den Behandlungsergebnissen bezogen aufdie führende Fachdisziplin kamen zu keinen signifikanten Unterschieden [9, 19]. Diese Ergebnisse legen nahe, dass der Unfallchirurg bei der Akutversorgung von Schwerverletzten durch standardisierte Systeme ersetzbar wäre. Dem entgegengesetzt sind Untersuchungen aus den USA, welche bessere Outcomes zeigten, wenn erfahrene Unfallchirurgen unmittelbar verfügbar waren [23].

\section{Damage control surgery}

Die Kenntnis des Damage-control-surgery(DCS)-Prinzips ist für den Traumateamleader unumgänglich, um ein gutes Behandlungsergebnis bei der Versorgung von Schwerverletzten zu erreichen [26, 33].

In der Unfallchirurgie sowie Orthopädie ist weltweit das DCS-Prinzip zentraler Ausbildungsinhalt und schon zu einem frühen Ausbildungsstand bekannt [12]. Dadurch sind Unfallchirurgen mit prioritätenbezogener Diagnostik und Therapie auf Basis von Schadensbegrenzung und "treat first what kills first" im Schockraum vertraut [17].

DCS beinhaltet die umfassende Beurteilung des zu erwartenden Outcomes unter Miteinbeziehung aller Faktoren. Für Patienten mit Polytrauma und SHT kann das den entscheidenden Unterschied zwischen einem Überleben mit möglichen neurologischen Defiziten oder dem Schicksal eines Apallikers bedeuten. Unter Anwendung der in der Ausbildung erlernten DCS-Prinzipien sind Unfallchirurgen gut ausgestattet, um eine Gesamtsituation schnell einschätzen zu können, Entscheidungen $\mathrm{zu}$ treffen und entsprechende Schritte einzuleiten.

\section{Geographischer Einfluss auf das Outcome}

In Australien gibt es aufgrund der geographischen Gegebenheiten mit großen Distanzen zwischen Krankenhäusern trotz regionalisiertem Strukturplan für das Transportwesen die Notwendigkeit der neurotraumatologischen Versorgung durch andere Fachdisziplinen als die der Neurochirurgie. Zahlreiche Studien untersuchten die Ergebnisse von Allgemeinchirurgen, die ein SHT operativ versorgten und konnten vergleichbare Ergebnisse für die Versorgung von Subdural- und Epiduralhämatomen zeigen $[20,29]$.

Leitlinien für das Management des Neurotraumas in ländlichen Regionen der Neurosurgical Society of Australasia empfehlen die selbstständige operative Versorgung durch alternative Fachärzte, wenn eine Zeitverzögerung von mehr als $2 \mathrm{~h}$ bis zu einer Versorgung durch Neurochirurgen zu erwarten ist [32]. Eine rezente schwedische Untersuchung der Ergebnisse neurotraumatologischer Operationen, welche aufgrund erwarteter langer Transportzeiten durch Allgemeinchirurgen durchgeführt wurden, ergab die Empfehlung, dieses Vorgehen beizubehalten [16].

\section{Einfluss von Ressourcen auf das Outcome}

Zahlreiche internationale Studien, insbesondere aus den USA, zeigen Versorgungsengpässe durch einen Mangel an Neurochirurgen und auch durch begrenzte Verfügbarkeit anderer Fachdisziplinen auf $[1,37]$.

Unter diesen Voraussetzungen entwickelte sich in den vergangenen Jahren in den USA die Fachdisziplin „acute care surgery“. Die Aufgaben des ,acute care surgeon" sind primär die hämodynamische Stabilisierung und das akute Management von Patienten mit allgemeinen sowie unfallchirurgischen Eingriffen [35]. Im Unterschied zum mitteleuropäischen Unfallchirurg wird ein großer Teil der Patienten von „acute care surgeons" nicht selbst operiert, sondern für andere Fachdisziplinen vorbereitet. Es gibt jedoch aufgrund der mangelhaften Verfügbarkeit von Fachärzten in den USA Stimmen, die ein größeres operatives Spektrum, inklusive Neurotraumatologie, für "acute care surgeons" einfordern [3, 6]. Damit zeichnet sich nach Studienlage in den USA aufgrund von Ressourcenmangel eine Entwicklung weg von „Spezialistentum“ zurück zum „Generalistentum“ ab. Gleichzeitig scheint aber das relativ junge Fach "acute care surgery" ein Image- und damit konsekutiv auch Nachwuchsproblem zu haben [31].

\section{1) Der "acute care surgeon“ sorgt für hämodynamische Stabilisierung und das akute Patientenmanagement}

Es ist davon auszugehen, dass auch in Mitteleuropa finanzielle Ressourcen für den Medizinsektor eher reduziert als ausgeweitet werden und in der Folge ein zunehmender Fachärztemangel bevorsteht. In Österreich besteht zum jetzigen Zeitpunkt ein Verhältnis von ca. 1600 Fachärzten für Unfallchirurgie zu ca. 190 Fachärzten für Neurochirurgie an 65 unfallchirurgischen und 11 neurochirurgischen Abteilungen. 
Interdisziplinäre Neurotraumatologie

Für die Versorgung des leichten und mittelschweren SHT sind Neurochirurgen meist nurkonsiliarisch in die Behandlung eingebunden und auch das konservative Management des schweren SHT wird oftmals nicht an neurochirurgischen Fachabteilungen durchgeführt [15].

In Österreich wird das SHT traditionell von Unfallchirurgen behandelt, wobei auch Kraniotomien, Bohrlochtrepanationen und die Implantation von Hirndrucksonden von Unfallchirurgen durchgeführt werden. Eine Studie von Drobetz et al. [11] zeigte, dass in Österreich $82 \%$ der Patienten mit schwerem SHT von unfallchirurgischen Abteilungen versorgt wurden. Bei einer noch nicht publizierten telefonischen Umfrage, welche durch die Autoren der vorliegenden Arbeit 2016 durchgeführt wurde, gaben nur noch $48 \%$ der 65 unfallchirurgischen Abteilungen in Österreich an, das schwere SHT auch operativ zu versorgen. Von diesen Abteilungen gaben weitere $12 \%$ an, nur im Notfall selbstständig zu operieren, sodass derzeit $36 \%$ der österreichischen Unfallchirurgien das schwere SHT selbstständig operativ versorgen. Weiter gab der Großteil der Abteilungen, die das schwere SHT nicht operieren, an, dass auch beim mittelschweren SHT routinemäßig eine teleradiologische Konsultation durch angebundene neurochirurgische Abteilungen erfolgt. Damit zeigt sich auch in Österreich, welches lange an der eigenständigen Unfallchirurgie festhielt und erst 2015 den Facharzt für Unfallchirurgie aufgab, ein Trend in Richtung interdisziplinärem Management des SHT, wobei die Abdeckung einer solchen Versorgungsstrategie nicht gewährleistet ist.

Es gibt nur wenige kontrollierte/ randomisierte Studien mit einem Vergleich zwischen neurotraumatologischer Versorgung und der Versorgung durch andere Fachdisziplinen. Für die sichere Implantation von Hirndrucksonden inklusive ventrikulärer Drainagen durch andere Fachdisziplinen als Neurochirurgen gibt es vergleichende Untersuchungen $[13,25]$. Darüber hinaus gehende operative Eingriffe durch alternative Fachärzte als Neurochirurgen wurden in Studien meist mit nationalen oder internationalen Ergebnissen verglichen und kamen mehrheitlich zu dem Schluss, dass die Ergebnisse vergleichbar sind [20, 29].

Die einzige prospektiv-vergleichende Studie wurde durch die International Neurotrauma Research Organization (INRO) multizentrisch in der Slowakei, Kroatien und Österreich durchgeführt. Der Vergleich der Ergebnisse von Kraniotomien durch Unfall- und Neurochirurgen bei isoliertem schwerem SHT zeigte keine signifikanten Unterschiede bzgl. der Mortalität. Auffallend war aber ein niedrigerer Glasgow Outcome Scale in der neurochirurgischen Gruppe, wobei diese Gruppe allerdings auch eine höhere Verletzungsschwere aufwies. Die Rate der postoperativen Nachblutungen war in der Gruppe der von Unfallchirurgen versorgten Patienten etwas höher, jedoch ohne signifikante Auswirkung auf die Mortalität. Insgesamt zeigten sich somit bei durch Unfallchirurgen und Neurochirurgen versorgtem SHT vergleichbare Resultate [27].

\section{Schlussfolgerungen}

Auch im Zeitalter der Standardisierung und des hohen Spezialisierungsgrads in der Medizin steht der Unfallchirurg im Zentrum der Versorgung beim Polytrauma mit begleitendem SHT. Die Kernkompetenz des Unfallchirurgen ist das Sicherstellen der prioritätenbezogenen Diagnostik und Therapie, denn dies erfordert breite Kenntnis und Fertigkeiten, welche in mitteleuropäischen Systemen mit einer gewachsenen allgemeinen Unfallchirurgie strukturell noch gegeben sind.

Die Grenzen der operativen Versorgung des Polytraumas und schweren SHT durch Unfallchirurgen werden auch weiterhin durch Systemfaktoren vorgegeben und unterstehen zudem zunehmend einem ressourcenabhängigen Wandel. Durch die Verschlechterung des Outcomes durch Zeitverzögerungen vor einer Operation wird auch in Zukunft eine operative Versorgung des schweren SHT durch zeitnah verfügbare Unfallchirurgen unumgänglich sein.
Will man vermeiden, dass in Zukunft ein potenzieller Mangel an Spezialisten zu einer Gefahr für die Versorgung schwer verletzter Patienten wird, heißt es, der Rolle des Unfallchirurgen beim Polytrauma und SHT entsprechendes Gewicht $\mathrm{zu}$ geben. Auf dieser Grundlage muss für eine adäquate Ausbildung zukünftiger Unfallchirurgen gesorgt und die Verantwortlichen in Politik und Standesvertretungen in die Pflicht genommen werden.

Die Autoren der vorliegenden Arbeit bekennen sich klar zu einer umfassenden unfallchirurgischen Akutversorgung von Patienten mit Polytrauma und SHT mit einer bedarfsorientierten fachüberschreitenden Zusammenarbeit im Sinne einer Sekundärversorgung, welche hinsichtlich des Behandlungsergebnisses auch vereinbar und sinnvoll ist.

\section{Fazit für die Praxis}

\section{- Hypotension ist die wichtigste Ein- flussgröße auf die Letalität des SHT beim Polytrauma. \\ - Der Traumateamleader muss die ent- scheidenden Outcomefaktoren zur Vermeidung eines sekundären Hirn- schadens kennen (Vitalparameter, Zeit). \\ - Kernkompetenz des Unfallchirurgen ist das Sicherstellen der prioritäten- bezogenen Diagnostik und Therapie sowie Einhaltung der Prinzipien der Damage Control Surgery. \\ - Neurotraumatologie kann von Un- fallchirurgen mit guten Ergebnissen praktiziert werden.}

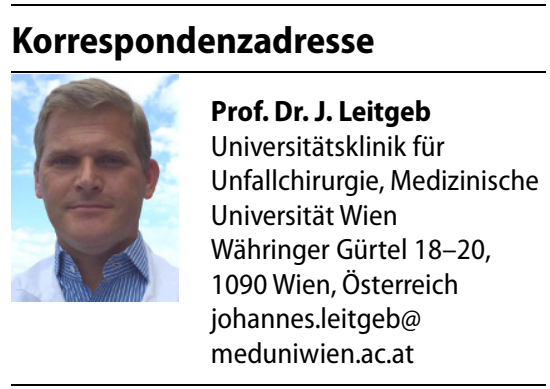

Open access funding provided by Medical University of Vienna. 


\section{Einhaltung ethischer Richtlinien}

Interessenkonflikt. A. Antoni, T. Heinz und J. Leitgeb geben an, dass kein Interessenkonflikt besteht.

Dieser Beitrag beinhaltet keine von den Autoren durchgeführten Studien an Menschen oder Tieren.

Open Access. Dieser Artikel wird unter der Creative Commons Namensnennung 4.0 International Lizenz (http://creativecommons.org/licenses/by/4.0/deed. de) veröffentlicht, welche die Nutzung, Vervielfältigung, Bearbeitung, Verbreitung und Wiedergabe in jeglichem Medium und Format erlaubt, sofern Sie den/die ursprünglichen Autor(en) und die Quelle ordnungsgemäß nennen, einen Link zur Creative Commons Lizenz beifügen und angeben, ob Änderungen vorgenommen wurden.

\section{Literatur}

1. Barratt $H$, Wilson M, Moore F, Raine R (2010) The implications of the NICE guidelines on neurosurgical management for all severe head injuries: systematic review. Emerg Med J 27(3):173-178

2. Brain Trauma Foundation, Bratton SL, Chestnut RM, Ghajar J, McConnell Hammond FF, Harris OA, Hartl R, Manley GT, Nemecek A, Newell DW, Rosenthal G, Schouten J, Shutter L, Timmons SD, Ullman JS, Videtta W, Wilberger JE, Wright DW (2007) Guidelines for the management of severe traumatic brain injury. I. Blood pressure and oxygenation. J Neurotrauma 24(Suppl 1):7-13

3. Byrne RW, Bagan BT, Bingaman W, Anderson VC, Selden NR (2011) Emergency neurosurgical care solutions: acute care surgery, regionalization, and the neurosurgeon: results of the 2008 CNS consensus session. Neurosurgery 68(4):1063-1067

4. Champion HR, Sacco WJ, Copes WS (1992) Improvement in outcome from trauma center care. Arch Surg 127(3):333-338

5. Chesnut RM, Marshall LF, Klauber MR, Blunt BA, Baldwin N, Eisenberg HM, Jane JA, Marmarou A, Foulkes MA (1993) The role of secondary brain injury in determining outcome from severe head injury. J Trauma 34(2):216-222

6. Ciesla DJ, Moore EE, Cothren CC, Johnson JL, Burch JM (2006) Has the trauma surgeon become house staff for the surgical subspecialist? Am J Surg 192(6):732-737

7. Committee on Trauma, American College of Surgeons (2014) Resources for optimal care of the injured patient. American College of Surgeons, Chicago

8. Cornwell EE 3rd, Chang DC, Phillips J, Campbell KA (2003) Enhanced trauma program commitment at a level I trauma center: effect on the process and outcome of care. Arch Surg 138(8):838-843

9. Cummings GE, Mayes DC (2007) A comparative study of designated trauma team leaders on trauma patient survival and emergency department length-of-stay.CJEM 9(2):105-110

10. Demetriades D, Murray J, Charalambides K, Alo K, Velmahos G, Rhee P, Chan L (2004) Trauma fatalities: time and location of hospital deaths. J Am Coll Surg 198(1):20-26

11. Drobetz $H$, Freudenschuss $B$, Kutscha-Lissberg E, Buchinger W, Mauritz W (2000) The treatment of severe head-brain injuries in Austria. Anasthesiol Intensivmed Notfallmed Schmerzther 35(10):630-634
12. Eardley WG, Taylor DM, Parker PJ (2010) Training in the practical application of damage control and early total care operative philosophy-perceptions of UK orthopaedic specialist trainees. Ann R Coll Surg Engl 92(2):154-158

13. Ekeh AP, llyas S, Saxe JM, Whitmill M, Parikh $P$, Schweitzer JS, McCarthy MC (2014) Successful placement of intracranial pressure monitors by trauma surgeons. J Trauma Acute Care Surg 76(2):286-290

14. El Mestoui Z, Jalalzadeh H, Giannakopoulos GF, Zuidema WP (2017) Incidence and etiology of mortality in polytrauma patients in a Dutch level I trauma center. Eur JEmerg Med 24(1):49-54

15. Esposito TJ, Reed RL 2nd, Gamelli RL, Luchette FA (2005) Neurosurgical coverage: essential, desired, or irrelevant for good patient care and trauma center status. Ann Surg 242(3):364-370

16. Fischerström A, Nyholm L, Lewén A, Enblad $P$ (2014) Acute neurosurgery for traumatic brain injury by general surgeons in Swedish county hospitals: a regional study. Acta Neurochir (Wien) 156(1):177-185

17. Frink M, Probst Ch, Krettek Ch, Pape HC (2007) Clinical management of polytraumatized patients in the emegergency room - duty and assignment of the trauma surgeon. Zentralbl Chir 132(1):49-53

18. Garwe T, Cowan LD, Neas B, Cathey T, Danford BC, Greenawalt $P(2010)$ Survival benefit of transfer to tertiary trauma centers for major trauma patients initially presenting to nontertiary trauma centers. Acad Emerg Med 17(11):1223-1232

19. Georgiou A, Lockey DJ (2010) The performance and assessment of hospital trauma teams. Scand J Trauma Resusc Emerg Med 18:66

20. Gilligan J, Reilly P, Pearce A, Taylor D (2017) Management of acute traumatic intracranial haematoma in rural and remote areas of Australia. ANZ J Surg 87(1-2):80-85

21. Gross T, Schüepp $M$, Attenberger $C$, Pargger $\mathrm{H}$, Amsler F (2012) Outcome in polytraumatized patients with and without brain injury. Acta Anaesthesiol Scand 56(9):1163-1174

22. Haas NP, Hoffmann RF, Mauch C, von Fournier C, Südkamp NP (1995) The management of polytraumatized patients in Germany. Clin Orthop Relat Res 318:25-35

23. HautER, Chang DC, Efron DT, CornwellEE 3rd (2006) Injured patients have lower mortality when treated by "full-time" trauma surgeons vs. surgeons who cover trauma "part-time".J Jrauma 61(2):272-278

24. Hukkelhoven CW, Steyerberg EW, Rampen AJ, Farace E, Habbema JD, Marshall LF, Murray GD, Maas Al (2003) Patient age and outcome following severe traumatic brain injury: an analysis of 5600 patients. J Neurosurg 99(4):666-673

25. Ko K, Conforti A (2003) Training protocol for intracranial pressure monitor placement by nonneurosurgeons: 5-year experience. J Trauma 55(3):480-483

26. Lehmann U, Rickels E, Krettek C (2001) Multiple trauma with craniocerebral trauma. Early definitive surgical management of long bone fractures? Unfallchirurg 104(3):196-209

27. Leitgeb J, Mauritz W, Brazinova A, Matula C, Majdan M, Wilbacher I, Rusnak M (2012) Outcome of patients with severe brain trauma who were treated either by neurosurgeons or by trauma surgeons. J Trauma Acute Care Surg 72(5):1263-1270

28. Leitgeb J, Mauritz W, Brazinova A, Majdan M, Wilbacher I (2013) Impact of concomitant injuries on outcomes after traumatic brain injury. Arch Orthop Trauma Surg 133(5):659-668
29. LuckT, Treacy PJ, Mathieson M, Sandilands J, Weidlich S, Read D (2015) Emergency neurosurgery in Darwin:still the generalist surgeons' responsibility. ANZ J Surg 85:610-614

30. McSwain N, Rotondo M, Meade P, Duchesne J (2012) A model for rural trauma care. Br J Surg 99(3):309-314

31. Montgomery SC, Privette AR, Ferguson PL, Mirdamadi M, Fakhry SM (2015) Inadequately marketing our brand: medical student awareness of acute care surgery. J Trauma Acute Care Surg 79:858-864

32. Neurosurgical Society of Australasia Inc (2009) The management of acute neurotrauma in rural and remote locations: a set of guidelines for the care of head and spinal injuries, 2. Aufl. Neurosurgery Society of Australasia Inc, East Melbourne

33. Pape HC, Giannoudis P, Krettek C (2002) The timing of fracture treatment in polytrauma patients: relevance of damage control orthopedic surgery. Am J Surg 183(6):622-629

34. Pfeifer R, Tarkin IS, Rocos B, Pape HC (2009) Patterns of mortality and causes of death in polytrauma patients - has anything changed? Injury 40(9):907-911

35. Pottenger BC, Galante JM, Wisner DH (2015) The modern acute care surgeon: characterization of an evolving surgical niche. J Trauma Acute Care Surg 78(1):120-125

36. Probst C, Pape HC, Hildebrand F, Regel G, Mahlke L, Giannoudis P, Krettek C, Grotz MR (2009) 30 years of polytrauma care: an analysis of the change in strategies and results of 4849 cases treated at a single institution. Injury 40(1):77-83

37. Rao MB, Lerro C, Gross CP (2010) The shortage of on-call surgical specialist coverage: a national survey of emergency department directors. Acad Emerg Med 17(12):1374-1382

38. Stone JL, Lowe RJ, Jonasson O, Baker RJ, Barrett J, Oldershaw JB, Crowell RM, Stein RJ (1986) Acute subdural hematoma: direct admission to a trauma center yields improved results. J Trauma 26:445-450

39. Wilberger JE Jr, Harris M, Diamond DL (1991) Acute subdural hematoma: morbidity, mortality, and operative timing. J Neurosurg 74:212-218 